\title{
Polymerase Chain Reaction (PCR)
}

National Cancer Institute ( $\mathrm{NCl})$

\section{Source}

National Cancer Institute (NCI). Polymerase chain reaction.

A procedure that produces millions of copies of a short segment of DNA through repeated cycles of: (1) denaturation, (2) annealing, and (3) elong ation. Polymerase chain reaction is a very common procedure in molecular genetic testing and may be used to generate a sufficient quantity of DNA to perform a test (e.g., allele-specific amplification, trinucleotide repeat quantification). Also called PCR. 